\title{
The Effects of Tissue Flossing on Perceived Knee Pain and Jump Performance: A Pilot Study
}

\author{
García-Luna Marco A., Cortell-Tormo Juan M.*, González-Martínez Julián, García-Jaén Miguel \\ Area of Physical Education and Sport, University of Alicante, Spain
}

Received January 12, 2020; Revised February 11, 2020; Accepted February 24, 2020

Copyright $\bigcirc 2020$ by authors, all rights reserved. Authors agree that this article remains permanently open access under the terms of the Creative Commons Attribution License 4.0 International License

\begin{abstract}
Background: Tissue compression and partial vascular occlusion using band flossing results in reperfusion of blood to the muscle tissue that may ultimately reduce joint pain and increase range of motion, enhancing prevention from or rehabilitation of injury. However, the extent of research examining the effect of tissue flossing in an athletic setting is currently very limited, and the effects of band flossing on knee pain and jump performance have not yet been investigated and remain unclear. Purpose: To investigate the effect of band flossing on perceived knee pain and vertical jump performance in recreational athletes with knee pain. Methods: Five young male recreational athletes with previously reported knee pain took part in this study (age $22 \pm 0.5$; height $184 \pm 6.8 \mathrm{~cm}$; weight $79 \pm 1.5 \mathrm{~kg}$; BMI $23.34 \pm 1.2 \mathrm{~kg} / \mathrm{m} 2$ ). Participants performed a number of tests pre and post intervention, with the application of a floss band on the knee joint. The experimental protocol consisted in the performance of countermovement jump (CMJ) tests (without occlusion, with occlusion, and after occlusion). Pre and Post intervention measures included a perceived knee pain -by visual analogue scale (VAS)- and CMJ performance -by force platform-. Results: The application of flossing bands in knee joint resulted in enhancements in all test measures pre to post intervention (10\% jump height; $4.5 \%$ time in the air; $5 \%$ jump velocity; $13 \%$ jump power; $7.5 \%$ jump force). Participants reported a 3.5-point pain enhancement in AVS during performance of CMJ tests. Conclusion: Flossing bands caused a reduction in perceived knee pain and improved vertical jump performance in young male recreational athletes.
\end{abstract}

Keywords Floss Bands, Vascular Occlusion, Joint Pain, Countermovement Jump, Patellofemoral

\section{Introduction}

In recent years, the use of compressive materials has grown considerably, both for the improvement of physical performance and for health. This boom is due, to a greater extent, to the benefits provided by their use. For its correct application, different types of garments and/or compressive materials have been developed that respond to different areas and objectives.

One of the most widespread compressive materials are compressive garments. Their origin comes from the health field and it has been demonstrated that they have positive effects on the blood circulation of the extremities [1,2]. In the field of sports, it has been proven that the prolonged use of compressive garments has improved performance in different sports [3-5]. The possible explanation would be its positive effects on venous hemodynamics [6], decreased exercise-induced muscle damage [7-9], and its possible contribution to recovery $[8,10-12]$. There are different types of compression garments, stockings, sleeves, upper body garments (covering the torso and upper limbs completely or partially) and lower body garments (from the waist, covering the lower limbs completely or partially) [13].

Another compressive material whose use has grown exponentially in recent years in the field of strength training, are occlusion cuffs. Unlike garments, these apply a band (inflated, elastic or rigid) to the area closest to the extremities with the intention of restricting the blood flow. Strength training with blood flow restriction (BFR) has shown significant gains in hypertrophy [14-16] and muscle strength [15,17-19]. In addition, these benefits are obtained by training at low intensity (loads around 20-40\% of $1 \mathrm{RM}$ ). It is therefore a very useful method for sectors of the population that, for health reasons, do not tolerate high loads on their joints and must improve their strength and hypertrophy [17,20-22].

On the other hand, recently a new material and method has been incorporated to compress the joints by means of an elastic band called flossing or floss band [23]. This consists of the use of reusable rubber bands with the aim of compressing a muscle region or a joint. Its purpose is to 
improve the range of motion (ROM), restore the mechanics of the joints and decompose the adhesive tissue of the previously injured musculature.

There is scientific evidence that supports its use to increase ROM [24-26] and pain reduction [24,27]. However, these results are not conclusive as there are other studies in which no improvement has been observed in these two variables $[28,29]$. Most studies have applied the flossing band to the ankle [25,26,30,31], the shoulder [29], or the wrist [24]. Only in one case has it been applied to the knee and improvements in strength were observed [32].

Concurrently, one of the most common knee problems is patellofemoral pain syndrome (PFPS), which is characterized by the presence of peripatellar or anterior knee pain [33], affecting one in four people [34,35], and also being responsible for a high percentage of knee sports injuries [36]. For that reason, it would be helpful to find a non-invasive way to improve this issue.

The effects of the flossing band on ROM, performance and knee pain are unknown, but since improvements have been seen in other joints [24,27], and other therapies similar to flossing band, such as bandaging techniques (Infrapatellar, Kinesiotape or McConnell), have been shown to help reduce painful symptoms and improve muscle function and strength [37-41], flossing band could probably also be applicable to the knee joint.

Therefore, the objective of this work is to investigate the effect of elastic bands (flossing) on perceived knee pain and vertical jump performance in amateur athletes with PFPS.

\section{Materials and Methods}

\subsection{Participants}

Five young male recreational athletes with PFPS took part in this study (age $=22.0 \pm 0.5$ years; height $=184.1 \pm 6.8$ $\mathrm{cm}$; weight $=79.0 \pm 1.5 \mathrm{~kg}$; BMI $=23.34 \pm 1.20 \mathrm{~kg} / \mathrm{m}^{2}$ ). To be eligible for this study, they all had to have been diagnosed with the PFPS for at least three months, but no known knee injury. They all had strength training experience and signed an informed consent form prior to any intervention or data collection. Candidates who could be adversely affected (cardiovascular or joint health) by the intervention in the study were excluded.

\subsection{Procedure}

The participants performed a pre and post test, with or without application of a floss band (Life Floss bands, Sydney, Australia) on the painful knee. Two different days (intervention and control) were used to carry out the protocol, using the application of the floss band in the first of them, and eliminating its use in the second. The aim of the separation into two days was to rule out that the application of one protocol would affect the results of the next.

On the first day (FLOSS) three series of three counter movement jumps (CMJ) were performed, with the average of the three jumps in each series. CMJ was performed from an upright position with straight legs, beginning the jump with a counter movement down to a knee angle of 90 degrees. Hands were held on the waist during the whole jump to avoid any effect of arm-swing. We used 1-minute rest between series, and 15 seconds between repetitions. 1st series: without application of floss band (PRE); 2nd series: with application of floss band (FB) (Figure 1); 3rd series: without application of floss band, after removal (POST). On the second day $(\mathrm{CON})$, the same protocol was performed, but without the application of floss band: three series (S1, S2 and S3) of three CMJ with 1-minute rest between series and 15 seconds between repetitions. The same jump performance and perceived pain variables were measured on both days.

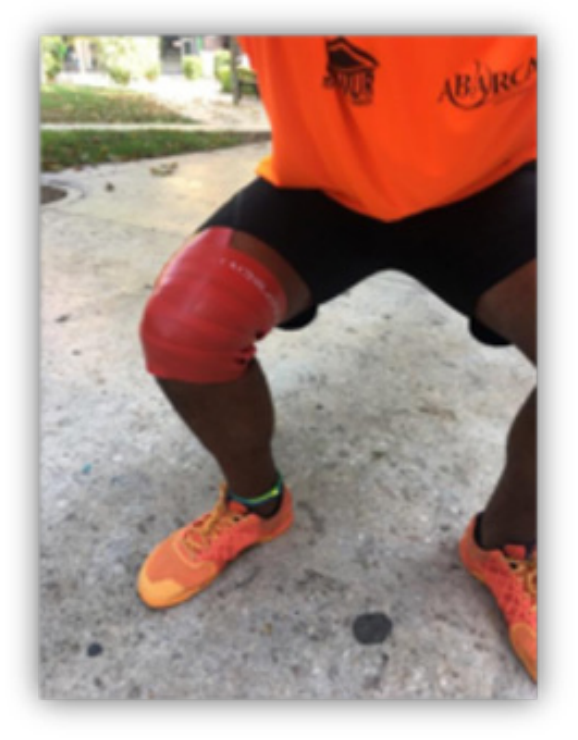

Figure 1. CMJ with floss band

\subsubsection{CMJ}

Each participant performed the CMJs with their hands on their waist, starting from an upright position and keeping their legs extended throughout the flight phase. Variable jump height, time in the air, jump velocity, jump power and applied force were measured using a force platform (Kistler 9287 BA, Kistler Instruments Ltd., Hook, UK).

Perceived knee pain was also measured in all three phases of the protocol. Each phase corresponds to the perceived pain at the end of each series of three CMJs. A visual analogue scale (VAS) divided into 10 equal parts (indicating 0 as no pain and 10 as unbearable pain) was used for assessment. 


\subsubsection{Floss Band Application}

The painful knee was bandaged according to the manufacturer, surrounding it from the most proximal to the most distal area, leaving $30 \%$ of the band visible and overlapping along the joint (Figure 2). To ensure that the pressure of the band was adequate, the Kikuhime pressure measuring sensor (MediGroup, Melbourne, Australia), located between the band and the lateral condyle of the femur, was used. The average pressure was $182 \pm 38$ $\mathrm{mmHg}$.

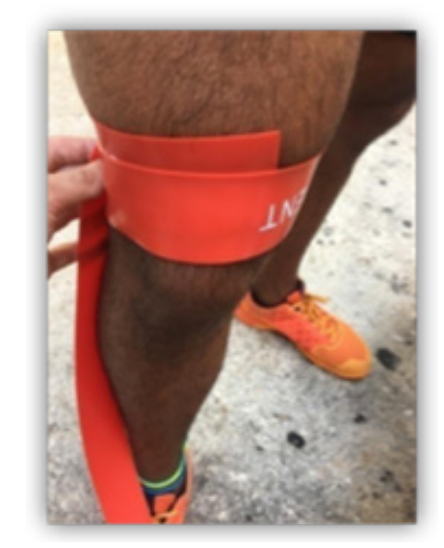

Figure 2. The floss band knee bandaging technique used

\subsection{Statistical Analysis}

Data analysis was performed using the IBM SPSS
Social Science Statistical Package, version 25.0 (SPSS Inc., Chicago, IL, USA). The sample distribution was tested using the Shapiro-Wilk test. A T-Student test was performed to check if there were significant differences. A value of $p<0.05$ was established to determine statistical significance.

\section{Results}

Significant differences were found in all the analysed variables regarding the performance of the vertical jump in the protocol applying the floss bands $(p<0.05)$. No significant differences were found in any of the variables analysed related to the vertical jump performance in the protocol that did not apply the floss bands $(p=0.283)$. Significant differences in perceived pain were found in the protocol applying the floss bands $(p<0.001)$. No significant difference in perceived pain was found in the protocol that did not apply floss bands $(\mathrm{p}=0.413)$. Table 1 presents these results.

All variables were evaluated during the three series of jumps, although significant differences were only found between the first and third series. Thus, the application of the floss bands was found to significantly improve the performance of the $\mathrm{CMJ}$, as well as significantly reduce the perception of pain in the knee where the floss bands were applied, but not during their application, but after their removal.

Table 1. Pre, FB and Post (or S1, S2, S3) measures (mean \pm SD) and percentage of variation, in the two days of intervention

\begin{tabular}{|c|c|c|c|c|c|c|c|c|}
\hline & \multicolumn{4}{|c}{ FLOSS (mean \pm SD) } & \multicolumn{4}{c|}{ CON (mean \pm SD) } \\
\hline & PRE & FB & POST & $\Delta(\%)$ & S1 & S2 & S3 & $\Delta(\%)$ \\
\hline JH $(\mathrm{cm})$ & $36.03 \pm 5.21$ & $36.39 \pm 7.09$ & $40.02 \pm 5.60$ & $11.1 \pm 1.4^{*}$ & $31.69 \pm 4.45$ & $31.46 \pm 5.12$ & $32.61 \pm 4.73$ & $2.9 \pm 0.8$ \\
\hline TA $(\mathrm{s})$ & $0.542 \pm 0.076$ & $0.549 \pm 0.081$ & $0.571 \pm 0.082$ & $5.4 \pm 0.7^{*}$ & $0.508 \pm 0.071$ & $0.498 \pm 0.079$ & $0.521 \pm 0.074$ & $2.6 \pm 0.5$ \\
\hline JV $(\mathrm{m} / \mathrm{s})$ & $1.33 \pm 0.19$ & $1.34 \pm 0.21$ & $1.41 \pm 0.20$ & $6.0 \pm 0.8^{*}$ & $1.25 \pm 0.18$ & $1.24 \pm 0.21$ & $1.28 \pm 0.18$ & $2.4 \pm 0.4$ \\
\hline JP $(\mathrm{W})$ & $3837 \pm 537$ & $3852 \pm 539$ & $4371 \pm 612$ & $13.9 \pm 1.8^{*}$ & $3280 \pm 460$ & $3257 \pm 441$ & $3389 \pm 497$ & $3.3 \pm 0.9$ \\
\hline JF $(\mathrm{N})$ & $2886 \pm 404$ & $2895 \pm 412$ & $3121 \pm 437$ & $8.1 \pm 1.1^{*}$ & $2630 \pm 368$ & $2614 \pm 349$ & $2697 \pm 386$ & $2.5 \pm 0.7$ \\
\hline AVS $1-10$ & $4.0 \pm 0.7$ & $3.8 \pm 0.5$ & $0.5 \pm 0.5$ & $-87.5 \pm 9.7^{* *}$ & $6.0 \pm 0.7$ & $5.5 \pm 0.8$ & $5.2 \pm 0.5$ & $-13.3 \pm 7.7$ \\
\hline
\end{tabular}

FLOSS $=$ Day when floss band protocol was applied; $\mathrm{CON}=\mathrm{Control}$ day in which the protocol was performed without the floss bands; $\mathrm{PRE}=$ Without application of floss band; FB= With application of floss band; POST= After removal of floss band; S1,S2,S3= Series 1,2,3; $\mathrm{JH}=\mathrm{Jump}$ height; $\mathrm{TA}=$ Time in the air; $\mathrm{JV}=\mathrm{Jump}$ velocity; $\mathrm{JP}=\mathrm{Jump}$ power; $\mathrm{JF}=\mathrm{Jump}$ force; AVS $(1-10)=$ Analogue visual scale.

${ }^{*}$ The differences are significant (Pre vs Post $\mathrm{p}<0.05$ ).

${ }^{* *}$ The differences are significant (Pre vs Post $\mathrm{p}<0.001$ ). 


\section{Discussion}

The aim of this work was to identify whether the application of the floss band to the knee joint could increase the performance of the $\mathrm{CMJ}$ and decrease the sensation of pain in this joint.

Different studies have shown the benefits of floss bands, although the vast majority have used them on joints other than the knee. Stevenson et al. [25] showed that, by compressing the ankle joint for 2 minutes, significant improvements were achieved in dorsiflexion of this joint $(p<0.032)$. Driller \& Overmayer [26], also found significant improvements in ankle ROM and one-leg jumping performance $(\mathrm{p}<0.01)$. The same research group in a later work found, in agreement with the previous one, that the application of the floss bands in both ankles during 2 minutes, could improve the performance in the ROM, the CMJ and the sprint until 45 minutes after its application [30]. However, although significant differences were found in ankle ROM $(\mathrm{p}<0.05)$, both $\mathrm{CMJ}$ and sprint performance were not $(p>0.05)$. Very similar results were obtained by Mills et al. [31], finding improvements in ankle ROM, $\mathrm{CMJ}$ and sprint performance, although not significant in the latter two $(\mathrm{p}<0.05)$.

Floss bands have also been applied to other joints, such as the shoulder, in order to analyse their influence on ROM and their power in the bench press movement [29]. Although no significant differences were found in any of these variables, the authors suggest that floss bands may have a greater influence on less complex joints than the shoulder. They attribute this lack of efficacy to the deficiency of the bandage to compress all the muscles involved in the movements evaluated. On the other hand, the wrist has also been analysed, in a case study based on a participant with "Kienböck disease" (aseptic avascular necrosis of the crescent carpal), who presented pain and swelling on the right wrist dorsum [24]. In this work, the influence of the floss band on pain reduction and improvement of wrist functionality in aspects of daily life, such as pain, numbness, tingling, ability to drive or work, was analysed by means of questionnaires. After 6 weeks of application of the floss band treatment (1-3 minutes of application before your rehabilitation exercises), significant improvements in both pain perception and wrist functionality were found $(\mathrm{p}<0.05)$.

Although the use of the floss band is recent, different modes of compression or bandaging have shown benefits for decades. Patellar taping, for example, showed a significant decrease in pain $(\mathrm{p}<0.005)$ and an increase in performance in the Star Excursion Balance Test in patients with PFPS [39]. Kuru et al. [41] concluded that Kinesio bands showed the same benefits as electrostimulation in patients with PFPS, in terms of pain reduction, improved ROM, strength, functional capacity and quality of life. It is relevant to mention that both works, contrary to our results, showed the benefits during the application of the band, while in the present work the improvements came after removing the floss band.

Mason et al. [37], on the other hand, also found benefits in pain reduction in patients with PFPS by means of infrapatellar taping, being applied continuously throughout the week. However, their results concluded that greater benefits are obtained when combined with quadriceps stretching and strengthening work. Similar results were evidenced by Paoloni et al. [40], where they suggested that a short period (14 days) of patellar taping and a subsequent exercise program was a good means of controlling long-term pain in participants with PFPS associated with muscle dysfunction.

Although it is complicated to allude to the physiological or mechanical mechanisms that could have favoured the changes described, it seems that our results are in the same direction as other previous work that has used this type of implement or some similar ones. Therefore, it is suggested that future research will be able to identify what these mechanisms are, as well as to provide more evidence and clarity to joint compression by means of floss bands.

\section{Practical Applications}

Other studies have shown the importance of good ankle dorsiflexion in shock absorption in the lower extremities when landing a jump [42], thus justifying the use, prior to exercise, of floss bands on the ankle [30]. Therefore, the previous results, as well as those obtained in this study suggest that there could be powerful and useful practical applications, considering the use of floss bands during the warm-up prior to training, or even competition. This would be a good method to reduce pain produced by PFPS, as well as to increase performance in vertical jumping. Any sport or physical activity in which vertical jumping is a performance factor could benefit greatly from this type of technique, although future research should establish which protocol produces the greatest gains in these variables.

\section{Conclusions}

The present study adds useful and interesting information to the novel field of floss bands, in relation to the reduction of knee pain in participants with PFPS and the increase in CMJ performance. Both variables have been improved after the application of these bands and their subsequent removal. These preliminary results can have a relevant impact in the sports setting, both recreational and competitive. 


\section{REFERENCES}

[1] O. Agu, G. Hamilton, and D. Baker, "Graduated compression stockings in the prevention of venous thromboembolism", Br. J. Surg., vol. 86, no. 8, pp. 9921004, Aug. 1999.

[2] S. D. Blair, D. D. Wright, C. M. Backhouse, E. Riddle, and C. N. McCollum, "Sustained compression and healing of chronic venous ulcers", BMJ, vol. 297, no. 6657, pp. 115961, Nov. 1988.

[3] A. Bringard, R. Denis, N. Belluye, and S. Perrey, "Effects of compression tights on calf muscle oxygenation and venous pooling during quiet resting in supine and standing positions", J. Sports Med. Phys. Fitness, vol. 46, no. 4, pp. 548-54, Dec. 2006.

[4] B. Doan et al., "Evaluation of a lower-body compression garment”, J. Sports Sci., vol. 21, no. 8, pp. 601-610, Jan. 2003.

[5] W. J. Kraemer et al., "Influence of a compression garment on repetitive power output production before and after different types of muscle fatigue", Sport. Med. Train. Rehabil., vol. 8, no. 2, pp. 163-184, Feb. 1998.

[6] E. O. Brizzio, R. Stemmer, J. de Simone, and C. Salvia, "Los efectos hemodinámicos de las medias terapéuticas de comprensión sobre el retorno venoso", Rev. Asoc. Méd. Argent, pp. 26-31, 1994.

[7] R. B. Armstrong, G. L. Warren, and J. A. Warren, "Mechanisms of Exercise-Induced Muscle Fibre Injury", Sport. Med., vol. 12, no. 3, pp. 184-207, Sep. 1991.

[8] W. J. Kraemer et al., "Influence of Compression Therapy on Symptoms Following Soft Tissue Injury from Maximal Eccentric Exercise", J. Orthop. Sport. Phys. Ther., vol. 31, no. 6, pp. 282-290, Jun. 2001.

[9] T. J. Noonan and W. E. Garrett, "Muscle Strain Injury: Diagnosis and Treatment", J. Am. Acad. Orthop. Surg., vol. 7, no. 4, pp. 262-269, Jul. 1999.

[10] M. J. Berry and R. G. McMurray, "Effects of graduated compression stockings on blood lactate following an exhaustive bout of exercise", Am. J. Phys. Med., vol. 66, no. 3, pp. 121-32, Jun. 1987.

[11] J.-C. Chatard, D. Atlaoui, J. Farjanel, F. Louisy, D. Rastel, and C.-Y. Guzennec, "Elastic stockings, performance and leg pain recovery in 63-year-old sportsmen”, Eur. J. Appl. Physiol., vol. 93, no. 3, pp. 347-352, Dec. 2004.

[12] D. Marqués-Jiménez, J. Calleja-González, I. Arratibel, A. Delextrat, and N. Terrados, "Are compression garments effective for the recovery of exercise-induced muscle damage? A systematic review with meta-analysis", Physiol. Behav., vol. 153, pp. 133-148, Jan. 2016.

[13] B. A. MacRae, J. D. Cotter, and R. M. Laing, "Compression Garments and Exercise”, Sport. Med., vol. 41, no. 10, pp. 815-843, Oct. 2011.

[14] C. Centner, P. Wiegel, A. Gollhofer, and D. König, "Effects of Blood Flow Restriction Training on Muscular Strength and Hypertrophy in Older Individuals: A Systematic Review and Meta-Analysis", Sport. Med., vol. 49, no. 1, pp. 95-108, Jan. 2019.

[15] H. C. Heitkamp, "Training with blood flow restriction. Mechanisms, gain in strength and safety", J. Sports Med. Phys. Fitness, vol. 55, no. 5, pp. 446-56, May 2015.

[16] J. Slysz, J. Stultz, and J. F. Burr, "The efficacy of blood flow restricted exercise: A systematic review \&amp; meta-analysis", J. Sci. Med. Sport, vol. 19, no. 8, pp. 669675, Aug. 2016.

[17] B. R. Scott, J. P. Loenneke, K. M. Slattery, and B. J. Dascombe, "Exercise with Blood Flow Restriction: An Updated Evidence-Based Approach for Enhanced Muscular Development”, Sport. Med., vol. 45, no. 3, pp. 313-325, Mar. 2015.

[18] S.-Y. Park, Y. S. Kwak, A. Harveson, J. C. Weavil, and K. E. Seo, "Low Intensity Resistance Exercise Training with Blood Flow Restriction: Insight into Cardiovascular Function, and Skeletal Muscle Hypertrophy in Humans", Korean J. Physiol. Pharmacol., vol. 19, no. 3, p. 191, May 2015.

[19] Z. K. Pope, J. M. Willardson, and B. J. Schoenfeld, "Exercise and Blood Flow Restriction", J. Strength Cond. Res., vol. 27, no. 10, pp. 2914-2926, Oct. 2013.

[20] J. P. Loenneke, R. S. Thiebaud, and T. Abe, "Does blood flow restriction result in skeletal muscle damage? A critical review of available evidence", Scand. J. Med. Sci. Sports, vol. 24, no. 6, pp. e415-422, Dec. 2014.

[21] J. P. Loenneke, G. J. Wilson, and J. M. Wilson, "A Mechanistic Approach to Blood Flow Occlusion”, Int. J. Sports Med., vol. 31, no. 01, pp. 1-4, Jan. 2010.

[22] B. R. Scott, J. P. Loenneke, K. M. Slattery, and B. J. Dascombe, "Blood flow restricted exercise for athletes: A review of available evidence", J. Sci. Med. Sport, vol. 19, no. 5, pp. 360-367, May 2016.

[23] K. Starrett and G. Cordoza, Becoming a supple leopard : the ultimate guide to resolving pain, preventing injury, and optimizing athletic performance. 2013.

[24] A. A. Arce-Esquivel, S. A. Cage, B. J Warner, and P. Stevenson, "Flossing bands to treat keinböck's disease in a collegiate men's basketball player: a case report", Int. Phys. Med. Rehabil. J., vol. 3, no. 2, Apr. 2018.

[25] P. J. Stevenson, R. K. Stevenson, and K. W. Duarte, “Acute Effects of The Voodoo Flossing Band on Ankle Range of Motion”, J. Med. Biomed. Appl. Sci., vol. 7, no. 6, pp. 244253, Jun. 2019

[26] M. W. Driller and R. G. Overmayer, "The effects of tissue flossing on ankle range of motion and jump performance", Phys. Ther. Sport, vol. 25, pp. 20-24, May 2017.

[27] R. Prill, R. Schulz, and S. Michel, "Tissue flossing: a new short-term compression therapy for reducing exercise-induced delayed-onset muscle soreness. A randomized, controlled and double-blind pilot crossover trial", J. Sports Med. Phys. Fitness, vol. 59, no. 5, pp. 861867, Apr. 2019.

[28] V. Gorny and T. Stöggl, "Einfluss von Flossing auf die Regenerationsfähigkeit der unteren Extremität nach 
Kraftausdauer-Belastungen”, Sport. · Sport., vol. 32, no. 01, pp. 55-60, Mar. 2018.

[29] D. Plocker, B. Wahlquist, and B. Dittrich, "Effects of tissue flossing on upper extremity range of motion and power", Int. J. Exerc. Sci. Conf. Proc., vol. 12, no. 1, Nov. 2015.

[30] M. Driller, K. Mackay, B. Mills, and F. Tavares, "Tissue flossing on ankle range of motion, jump and sprint performance: A follow-up study", Phys. Ther. Sport, vol. 28, pp. 29-33, Nov. 2017.

[31] B. Mills, B. Mayo, F. Tavares, and M. Driller, "The Effect of Tissue Flossing on Ankle Range of Motion, Jump, and Sprint Performance in Elite Rugby Union Athletes", J. Sport Rehabil., pp. 1-5, Jun. 2019.

[32] J. Bohlen, M. Arsenault, B. Deane, P. Miller, M. Guadagno, and D. A. Dobrosielski, "Effects of Applying Floss Bands on Regional Blood Flow", Int. J. Exerc. Sci. Conf. Proc., vol. 9, no. 2, May 2014.

[33] J. H. L. Keet, J. Gray, Y. Harley, and M. I. Lambert, "The effect of medial patellar taping on pain, strength and neuromuscular recruitment in subjects with and without patellofemoral pain", Physiotherapy, vol. 93, no. 1, pp. 4552, Mar. 2007.

[34] J. McConnell, "The Management of Chondromalacia Patellae: A Long Term Solution", Aust. J. Physiother., vol. 32, no. 4, pp. 215-223, 1986.

[35] E. Witvrouw, R. Lysens, J. Bellemans, D. Cambier, and G. Vanderstraeten, "Intrinsic Risk Factors for the Development of Anterior Knee Pain in an Athletic Population: A Two-Year Prospective Study", Am. J. Sports Med., vol. 28, no. 4, pp. 480-489, Jul. 2000.

[36] J. E. Earl and C. S. Vetter, "Patellofemoral Pain", Phys. Med. Rehabil. Clin. N. Am., vol. 18, no. 3, pp. 439-458, Aug. 2007.

[37] M. Mason, S. L. Keays, and P. A. Newcombe, "The Effect of Taping, Quadriceps Strengthening and Stretching Prescribed Separately or Combined on Patellofemoral Pain", Physiother. Res. Int., vol. 16, no. 2, pp. 109-119, Jun. 2011.

[38] J. A. Osorio et al., "The effects of two therapeutic patellofemoral taping techniques on strength, endurance, and pain responses", Phys. Ther. Sport, vol. 14, no. 4, pp. 199-206, Nov. 2013.

[39] N. Aminaka and P. A. Gribble, "Patellar Taping, Patellofemoral Pain Syndrome, Lower Extremity Kinematics, and Dynamic Postural Control”, J. Athl. Train., vol. 43, no. 1, pp. 21-28, Jan. 2008.

[40] M. Paoloni, G. Fratocchi, M. Mangone, M. Murgia, V. Santilli, and A. Cacchio, "Long-term efficacy of a short period of taping followed by an exercise program in a cohort of patients with patellofemoral pain syndrome", Clin. Rheumatol., vol. 31, no. 3, pp. 535-539, Mar. 2012.

[41] T. Kuru, A. Yalıman, and E. E. Dereli, "Comparison of efficiency of Kinesio ${ }^{\circledR}$ taping and electrical stimulation in patients with patellofemoral pain syndrome", Acta Orthop. Traumatol. Turc., vol. 46, no. 5, pp. 385-92, 2012.

[42] P. Malliaras, J. L. Cook, and P. Kent, "Reduced ankle dorsiflexion range may increase the risk of patellar tendon injury among volleyball players", J. Sci. Med. Sport, vol. 9, no. 4, pp. 304-309, Aug. 2006. 